\title{
MiR-181c inhibits prostatic epithelial cell proliferation caused by chronic non-bacterial prostatitis through down- regulating $\mathrm{COX}-2$
}

\author{
$\mathrm{Xu} \mathrm{Xu} \mathrm{a}^{\mathrm{a}}$, Yuhua Huang ${ }^{\mathrm{a}}$, Jianquan Hou ${ }^{\mathrm{a}}$, Jinxing $\mathrm{Lv}^{\mathrm{b}}$, Xiang Ding, ${ }^{\mathrm{a},}$ \\ ${ }^{a}$ Department of Urology, The First Affiliated Hospital of Soochow University, Suzhou 215006, China. \\ ${ }^{b}$ Reproductive Medicine Center, The First Affiliated Hospital of Soochow University, Suzhou 215006, China.
}

\begin{abstract}
Background: Chronic non-bacterial prostatitis (CNP) is a widespread disease of the male reproductive system. MiR-181c can be expressed in prostate tissue, but it has not been reported in CNP. This study aims to investigate the role of miR-181c in CNP and its mechanism of action on CNP, providing new ideas for the treatment and diagnosis of CNP.

Methods: Quantitative real-time polymerase chain reaction (qRT-PCR) and western blotting were applied to determine miR-181c expression in clinical CP patients, CNP rats, and LPS-induced human prostaglandin epithelial cell RWPE-1. Then, luciferase reporter assay was performed to verify the targeting relation between miR-181c and COX-2. Through cell transfection experiments, the effect of mi-181c on the expression of COX2 and PGE2 was studied, and the effect of miR-181c/COX-2 on the proliferation of prostate epithelial cells was also explored.

Results: qRT-PCR and Western blotting analysis revealed that miR-181c was low expressed in prostate tissue of CP patients and CNP rats and human prostaglandin epithelial cell RWPE-1. The luciferase reporter assay confirmed the targeting relation between miR-181c and COX-2. And miR-181c overexpression reduced the expression of COX-2 and PGE2 and suppressed the proliferation of prostate epithelial cells. COX-2 up-regulation reversed these effects caused by overexpression of miR-181c.

Conclusions: miR-181c inhibited the proliferation of prostate epithelial cells through negatively regulating COX-2 to alleviate chronic non-bacterial prostatitis.
\end{abstract}

Keywords: Chronic non-bacterial prostatitis, miR-181c, COX-2, prostatic epithelial cell, proliferation

\section{Introduction}

Chronic prostatitis (CP) is one of the most common diseases in andrology, which often occurs in men at any age. In addition, men with a history of prostatitis present high recurrence rate with age, which is $20 \%, 38 \%$, and $50 \%$ in men aged 40,60, and 80, respectively [1]. According to the classification of National Institutes of Health $(\mathrm{NIH})$, prostatitis is sorted into the following categories: category I (acute bacterial prostatitis), category II (chronic bacterial

\footnotetext{
* Corresponding author: Xiang Ding

Mailing address: Department of Urology, The First Affiliated Hospital of Soochow University, No.188 Shizi Rd, Gusu District, Suzhou 215006, China.

Email: 18351188766@sina.cn

Received: 9 November 2020 / Accepted: 14 December 2020
}

prostatitis), category III (chronic prostatitis (CP)/chronic pelvic pain syndrome (CPPS)) and category IV (asymptomatic prostatitis). Category III is further classified into IIIA and IIIB. Category IIIA encompasses chronic nonbacterial prostatitis (CNP) [2]. Among them, CNP represents around $70-90 \%$ of patients diagnosed with prostatitis. The proportion is also increasing in recent years [3, 4]. The main clinical manifestations of CNP $[5,6]$ are irritation of the urethra and pain in the perineum. The etiology involves one or more factors such as body inflammation, endocrine, immune, and nerves. They act on prostate tissue alone or together to cause sensitization and neuroendocrine disruptions around the prostate, ultimately leading to uncontrolled regulation of the pelvic nerve by the cerebral cortex and chronic neuropathic pain $[7,8]$. To date, the pathogenesis of CNP has not been fully clarified, and the diagnosis and treatment of CNP a particular challenge.

Cyclooxygenase (COX), also called prostaglandin-endoperoxide $\mathrm{H}$ synthase (PGHS), is a rate-limiting enzyme 
that plays a vital role in prostaglandin (PG) biosynthesis from arachidonic acid and participates in diverse pathophysiological processes $[9,10]$. There are three different isoforms of COX: COX-1, COX-2, and COX-3. COX-1 serves a significant role in maintaining normal physiological functions, such as gastrointestinal mucosal protection and renal blood flow regulation. It is generally believed that COX-3 is a variant of COX-1, mainly in the cerebral cortex and heart. COX-2 is an inducible enzyme, which is either absent or expressed slightly in most cells [11]. However, when cells are stimulated by pro-inflammatory cytokines or cancer-promoting factors, $\mathrm{COX}-2$ shows upregulation and participates in inflammation as well as the formation and development of tumors $[12,13]$. Studies have shown that COX-2 is involved in CP [14, 15], and inhibition of COX-2 suppresses the proliferation of prostatic epithelial cells in CNP [16].

MicroRNA (miRNA) is a class of endogenous non-coding small RNA with 19-25 nt in length, which is relatively conserved during evolution. Lee et al. [17] found it for the first time in the genetic analysis of mutants of C. elegans (Clekgans) in 1993. Since miRNA was first discovered, the role of miRNA in disease has been a research hotspot in recent years. And many investigators have explored the association between miRNAs and male urogenital system diseases [18-20]. miR-181c is one of the important members of miRNA family. Overexpression of miR-181c can lead to mitochondrial complex IV remodeling and dysfunction [21], and increase the production of reactive oxygen species. There is a study indicating that miR-181c is down-regulated in prostate cancer cells [22]. However, miR-181c has not been reported in CNP. Interestingly, we found that miR-181c has a binding site with COX2 through the bioinformatics software microRNA.org. Therefore, we speculate that miR-181c may be one of the possible molecules regulating COX-2 in CNP.

In this study, we first examined miR-181c expression in patients with CP and LPS-induced human prostate epithelial cell line RWPE-1. Further studies verified the relationship between miR-181c and COX-2, and its effect on the proliferation of prostate epithelial cells. These findings may offer a potential candidate target for the therapeutic intervention of CNP, and an understanding of miR-181c in CNP by regulating COX-2.

\section{Materials and methods}

\section{Clinical sample}

Prostate tissue samples were obtained from Department of Urology of The First Affiliated Hospital of Soochow University, where 15 are from patients with CNP and 15 are from healthy male volunteers. The subjects were between 55 and 75 years old. The study was based on the Declaration of Helsinki of 1975. All men underwent prostate ultrasound-guided biopsy (transrectal ultrasoundguided biopsy TRUSBx) [23]. Prostate tissue samples were homogenized with a tissue homogenizer accord- ing to the manufacturer's instruction. In simple, tissues were weighed and minced with a scalpel blade. Then, the minced tissue samples were homogenized with cold homogenization buffer ( $100 \mathrm{mM}$ potassium phosphate, 0.25 M sucrose, PH 7.4) at $6000 \mathrm{rpm}$. Finally, the homogenized solution was transferred to a pre-cooled Eppendorf (EP) tube and stored at $-80^{\circ} \mathrm{C}$ until use.

\section{Quantitative real-time PCR (qRT-PCR).}

The total RNA in prostate tissues or RWPE-1 cells was extracted according to the TRIZOL method. miR-181c specific primers were used for the reverse transcription reaction of miRNA, and U6 was used as the internal reference. Simultaneously, $\beta$-actin served as an internal reference for the reverse transcription reaction of COX-2. RNA was transformed into cDNA with the reverse transcription kit (Qiagen). PCR amplification (based on each detected gene) was carried out with SYBR RT-PCR Kit (TaKaRa). Three specimens were set for each sample and the detected gene. The expression level of miR-181c and COX2 was calculated according to the formula $2^{-\Delta \Delta C \mathrm{~T}}$ method.

\section{Western blotting}

Prostate tissues or RWPE-1 cells were lysed using RIPA lysis solution (Beyotime). A BCA protein quantification kit (Boster) was applied to examine protein concentration. The protein sample was denatured with loading buffer, and then $20 \mu \mathrm{g}$ protein was separated on a $10 \%$ SDSPAGE gel. Then, the protein on the gel was transferred to a PVDF membrane. The membrane was blocked with 5\% BSA and incubated with primary antibodies, including anti-COX-2 and anti- $\beta$-actin (diluted 1:1,000) overnight at $4^{\circ} \mathrm{C}$. The next day, the membrane was incubated with goat anti-rabbit/mouse secondary antibody for $1 \mathrm{~h}$ at room temperature. Finally, ECL chemiluminescence reagent was used to quantify the relative expression of target protein, and Image J software (National Institutes of Health) was applied to analyze the protein bands.

\section{Cell culture}

Immortalized human prostate epithelial cell line RWPE1 cells were obtained from American type culture specimens (ATCC). The cells were incubated in keratinocyte serum-free medium (K-SFM) containing $50 \mu \mathrm{g} / \mathrm{mL}$ of bovine pituitary extract (BPE), 1\% of penicillin/streptomycin/amphotericin $\mathrm{B}$, and $5 \mathrm{ng} / \mathrm{mL}$ of epidermal growth factor. The cell culture flask is placed at $37^{\circ} \mathrm{C}$ with $5 \%$ carbon dioxide. Cells were routinely passaged at $80-90 \%$ confluence. RWPE-1 cells were stimulated with Lipopolysaccharide (LPS) solution $(10 \mu \mathrm{g} / \mathrm{mL})$ for $24 \mathrm{~h}$ and then tested for corresponding indicators.

\section{Cell apoptosis}

Flow cytometry was applied to analyze cell apoptosis. RWPE- 1 cells $\left(1 \times 10^{6}\right.$ cells/well $)$ were inoculated in a 6-well plate. After LPS treatment, cells were washed with PBS. The treated and untreated cells were resuspended in $100 \mu \mathrm{L}$ of binding buffer and then stained with $100 \mu \mathrm{L}$ of 
annexin $\mathrm{V}$ reagent and $5 \mu \mathrm{L}$ of PI for $15 \mathrm{~min}$ in the dark. A FACScan flow cytometer was applied to measure cell apoptosis according to the manufacturer' $s$ instruction. The data were analyzed by FlowJo (FlowJo, Ashland, OR, USA).

\section{Cell proliferation}

Cell proliferation was examined by a Cell-Light EdU DNA Cell Proliferation Kit (RiboBio) and MTT detection kit (Fluda) according to the manufacturer's instruction. RWPE-1 cells were inoculated on a 96-well plate. After LPS stimulation for $24 \mathrm{~h}$, cells were incubated with EdU solution for $2 \mathrm{~h}$. The cells were then fixed with $4 \%$ paraformaldehyde for $30 \mathrm{~min}$ and stained with Apollo Dye Solution. The nucleic acids were stained with Hoechst 33342. The rate of cell proliferation was calculated according to manufacturer's instruction. Images were collected by an IX71 fluorescence microscope (Olympus). For MTT assay, RWPE-1 cells were incubated in the cell incubator for 24 hours. The medium in each well was aspirated before measuring cell viability. And fresh RPMI medium containing $10 \% \mathrm{FBS}$ and $10 \mu \mathrm{L}$ of $0.5 \%$ MTT was added. Cells were cultured for another $4 \mathrm{~h}$. DMSO $(150 \mu \mathrm{L})$ was added to each well. The plate oscillated in a shaker for $10 \mathrm{~min}$. The absorbance was detected using a microplate reader at $490 \mathrm{~nm}$.

\section{Cell transfection}

RWPE-1 cells were cultured in a 24 -well plates for $24 \mathrm{~h}$. miR-181c inhibitor or miR-181c mimic was obtained from RiboBio. The COX-2 overexpression vector ( $\mathrm{pcD}$ NA-COX-2) and its negative control vector (pcDNA) were constructed by GeneChem (Shanghai, China). LipofectamineTM 2000 (Invitrogen, Carlsbad, California) was applied for cell transfection. Cells were collected for related analysis $48 \mathrm{~h}$ after transfection.

\section{Luciferase reporter assay}

Wild-type (WT) 3'-UTR COX-2 and mutant-type (Mut) 3'UTR COX-2 was constructed and cloned into the pcDNA empty vector. RWPE-1 cells were co-transfected with WT or Mut 3'-UTR COX-2 vector and miR-181c inhibitor or miR-181c mimic by LipofectamineTM 2000 reagent (Invitrogen). Firefly luciferase activity and Renilla luciferase activity were detected with a Dual-Luciferase Reporter Analysis Kit (Promega) according to the manufacturer's instruction. The relative luciferase activity of each transfected group was tested 3 times. The ratio of firefly luciferase to Renilla luciferase was used as the relative luciferase activity.e

\section{Enzyme-linked immunosorbent assay (ELISA)}

The supernatant of RWPE-1 cells was collected and centrifuged $(3,000 \mathrm{r} / \mathrm{min})$ at $4{ }^{\circ} \mathrm{C}$. The protein level of PEG2 was determined by an ELISA kit according to the manufacture's instruction. The detection was carried out by a multifunctional enzyme marker. The absorbance was determined at a wavelength of $450 \mathrm{~nm}$.

\section{Carrageenan-Induced CNP rats}

A total of 24 male Sprague Dawley SD rats (4-6 weeks old, 240-260 g) were obtained from Shanghai Experimental Animal Center. Animals were divided into 4 groups $(\mathrm{n}=6)$ : Sham, CNP, CNP+pre-NC, CNP+miR-181c mimic group. The animal experiments was carried out following the ethical guidelines and approved by the Institutional Animal Ethics Committee of The First Affiliated Hospital of Soochow University.

Each rat was injected with $1 \%$ carrageenan $(20 \mu \mathrm{L})$ and lentivirus $\left(5 \times 10^{7} \mathrm{TU} / \mathrm{mL}\right)$ carrying miR-181c mimic into the prostate under anesthesia. The Sham and CNP+pre$\mathrm{NC}$ group were given the same amount of normal saline or lentivirus carrying pre-NC. Seven days after the operation, the locomotion score was performed. During the observation period, 5 points were assigned when the hind limbs were completely bent or immobile. 1 point was assigned when there was no significant change in exercise capacity from the situation before the injection. All experiments were conducted by blind observers.

After that, the rats were sacrificed and prostate tissue was taken. One part was used to measure prostate volume, the rest was saved for later use. Six points were randomly counted in a high power field at 400 times magnification. And the inflammatory cells were quantified as the density per unit area. Two different histological sections of each rat were randomly selected from 20 microscope fields under a 6400-fold microscope to observe PCNA-positive cells in the ventral prostate epithelium of rat. Cell proliferation index was calculated based on the number of positive nuclei divided by the number of total nuclei, which expressed as a percentage. At least 2,000 cells per group were counted [24].

\section{Statistical analysis}

Student's t-test was applied to compare the difference $(p<0.05)$ of the data between two groups. A one-way analysis of variance (ANOVA) followed by the NewmanKeuls post hoc test was applied to evaluate the statistical significance $(p<0.05)$ between different groups.

\section{Results}

\section{miR-181c expression was down-regulated in CP patients}

To explore miR-181c expression in $\mathrm{CP}$, we selected healthy male volunteers and clinical CP patients. The expression of miR-181c and COX-2 was examined respectively, and the correlation between the two was analyzed. Our results indicated that miR-181c was lowly expressed in the prostate tissues of CP patients, while COX-2 was highly expressed in the prostate tissues (Figure 1A). Person correlation analysis showed that miR-181c and COX-2 had a negative correlation (Figure 1B).

\footnotetext{
miR-181c was down-regulated in LPS induced RWPE-1 cells
} 
Later, to further explore miR-181c expression in $\mathrm{CP}$, the expression of miR-181c and COX-2 was examined in LPSinduced RWPE-1 cells. The expression of miR-181c in RWPE-1cells in the LPS group was decreased compared with the control group (Figure 2A), while the expression of COX-2 mRNA and protein was increased (Figure 2B). We next performed flow cytometry to detect cell apoptosis. The results showed that RWPE-1 cell apoptosis was reduced after LPS treatment (Figure 2C). And the results of EdU assay indicated that LPS treatment increased the number of EdU positive cells (Figure 2D).

\section{Targeting relationship between miR-181c and COX-2}

Bioinformatics software microRNA.org was applied to predict the potential binding site between miR-181c and COX-2 (Figure 3C). To further explore the interaction between miR-181c and COX-2, a luciferase reporter assay was performed. Wild-type (WT) 3'-UTR COX-2 and mutant-type (Mut) 3'-UTR COX-2, miR-181c inhibitor, or miR-181c mimic were co-transfected into RWPE-1 cells. The results found that miR-181c expression was decreased after interference with miR-181c in COX-2 3'UTR-WT transfected RWPE-1 cells, while the luciferase activity of cells was increased, and the level of COX-2 mRNA and protein was also increased (Figure 3A). miR-181c was up-regulated in RWPE-1 cells transfected with COX-2 3'UTR-WT and miR-181c mimic, while the luciferase activity was decreased, and the level of COX-2 mRNA and
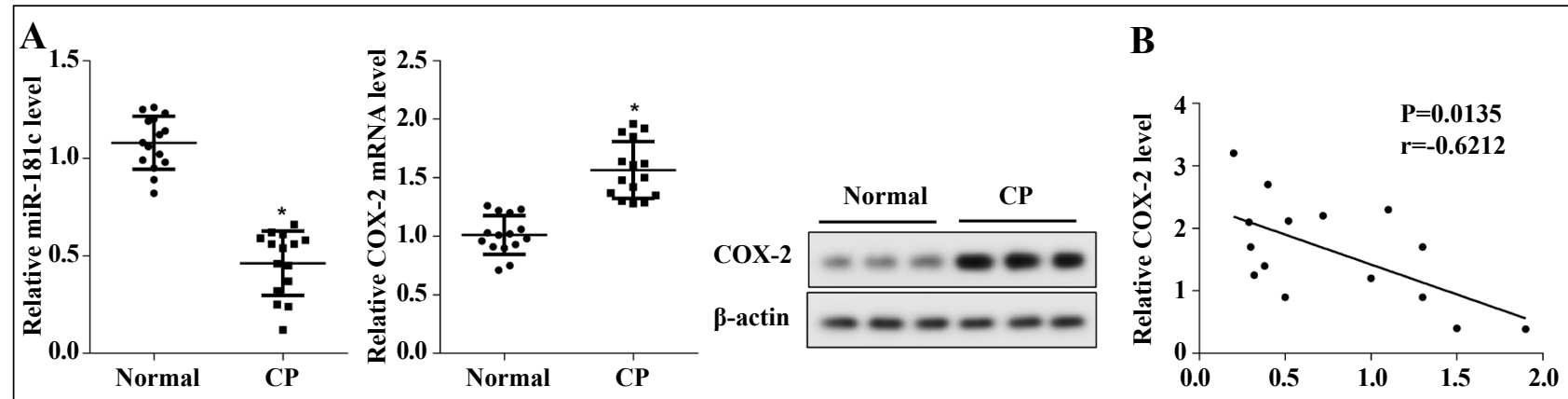

Figure 1. miR-181c was lowly expressed in prostate tissues of patients with CP. (A) The expression of miR-181c and COX-2 was measured by qRTPCR. The expression of COX-2 protein was examined by western blotting. (B) Person correlation was applied to examine the correlation between miR-181c and COX-2. $* \mathrm{P}<0.05$ vs. the normal group.
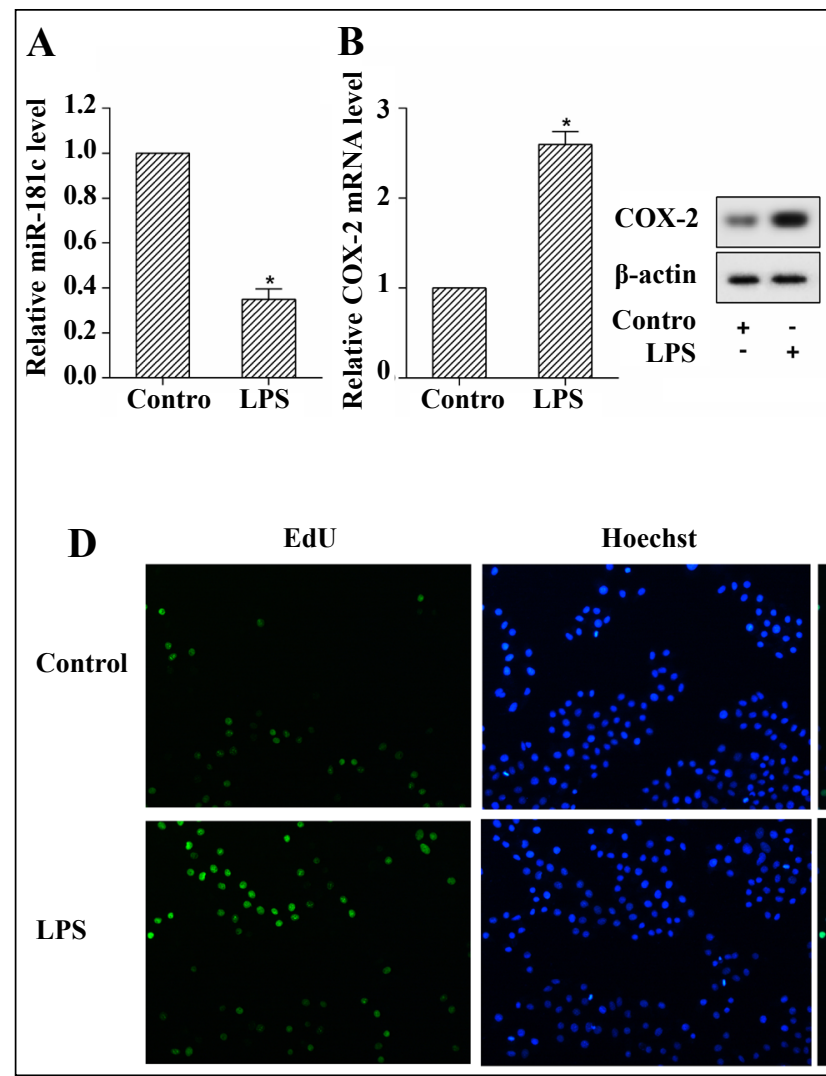

C
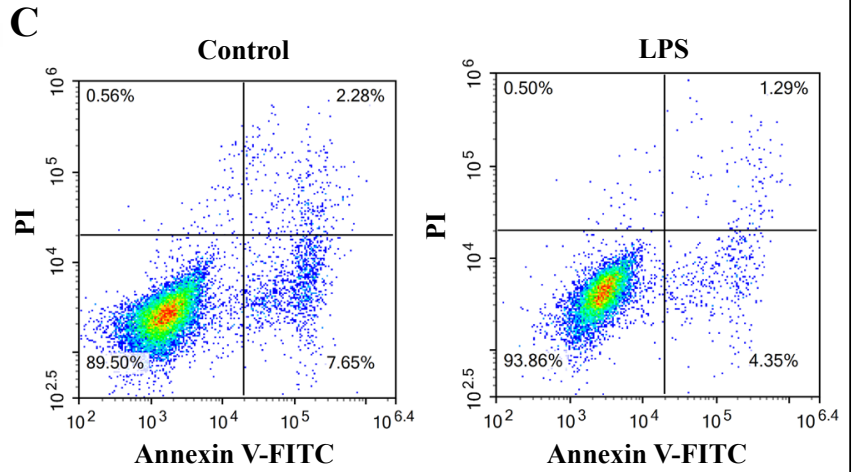

Figure 2. Human prostate epithelial cells RWPE-1 were isolated and cultured in vitro. LPS $(10 \mu \mathrm{g} / \mathrm{mL})$ was used to treat RWPE-1 cells. Cells were grouped as follows: control group and the LPS group. (A) miR-181c expression was analyzed by qRT-PCR. (B) qRT-PCR or western blotting was applied to detect COX-2 expression. (C) Cell apoptosis was detected using flow cytometry. (D) EdU assay was employed to examine cell proliferation. ${ }^{*} \mathrm{P}<0.05$ vs. the control group. 


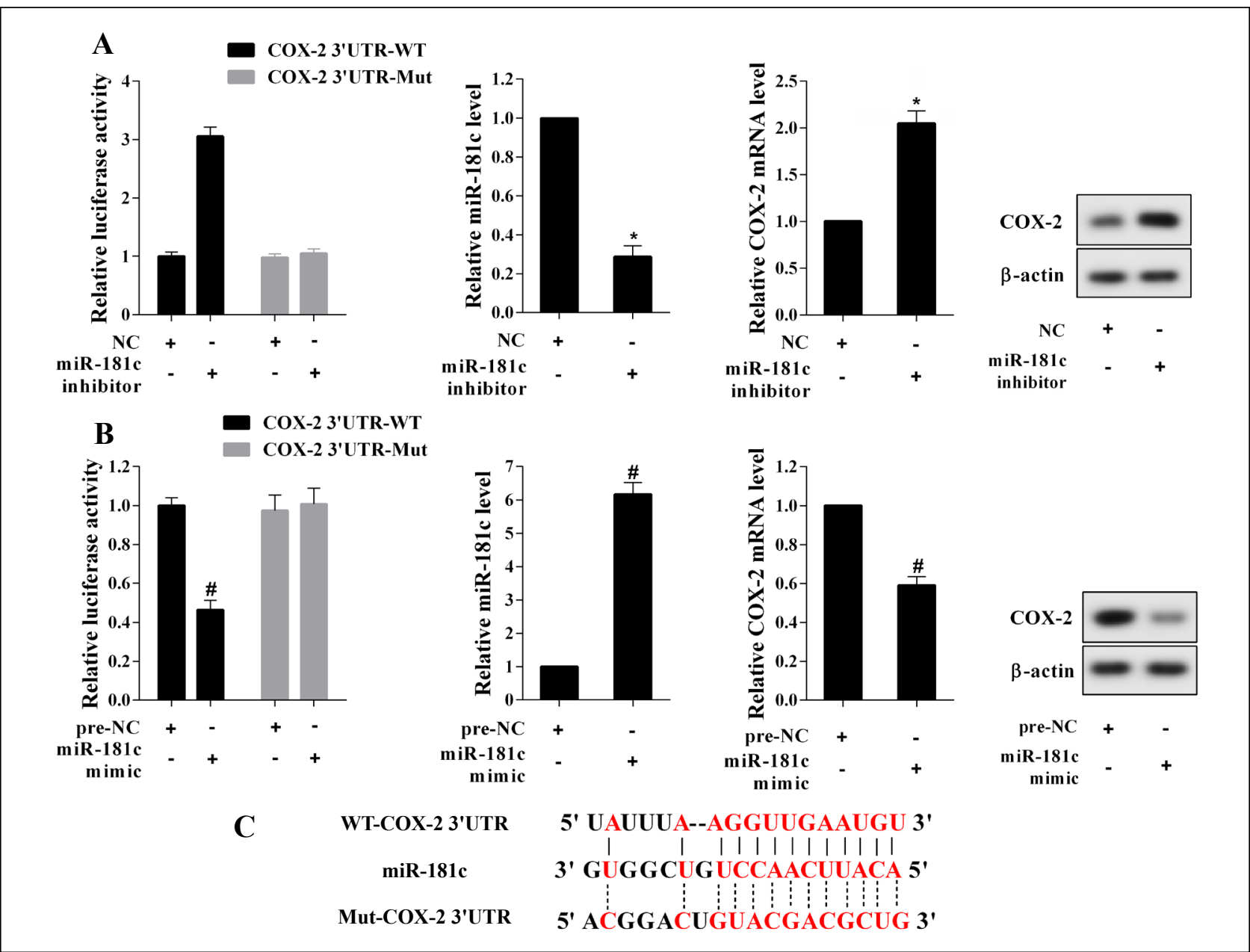

Figure 3. The targeting relationship between miR-181c and COX-2. Wild-type (WT) 3'-UTR COX-2, mutant-type (Mut) 3'-UTR COX-2, miR-181c inhibitor, or miR-181 c mimic were transfected into RWPE-1 cells, respectively. (A) The luciferase activity of RWPE-1 cells was measured using luciferase reporter assay, and qRT-PCR or western blotting was employed to measure the expression of miR-181c and COX-2 in RWPE-1 cells. (B) The luciferase activity of RWPE-1 cells was measured using luciferase reporter assay and qRT-PCR or western blotting was employed to measure the expression of miR-181c and COX-2 in RWPE-1 cells. (C) The binding site was predicted with microRNA.org. $* \mathrm{P}<0.05$ vs. the NC group, $\# \mathrm{P}<0.05$ vs. the pre-NC group.

protein were down-regulated (Figure 3B).

\section{Overexpression of miR-181c downregulated COX-2 ex- pression in LPS-induced RWPE-1 cells}

To further confirm the regulatory effect of miR-181c on COX-2, miR-181c mimic or pre-NC was transfected into RWPE-1 cells. QRT-PCR analysis indicated that miR$181 \mathrm{c}$ expression was increased in the LPS + miR-181c mimic group, indicating successful transfection (Figure $4 \mathrm{~A})$. The expression of COX-2 mRNA and protein were down-regulated after transfection of miR-181c mimic (Figure 4B), and the expression of its downstream synthetic product PGE2 was also decreased (Figure 4C).

Overexpression of COX-2 reversed miR-181c induced proliferation inhibition of prostate epithelial cells RWPE-1

RWPE-1 cells were transfected with miR-181c mimic or pcDNA-COX-2 to explore the effect of miR-181c/COX-2 on the proliferation of prostate epithelial cells. The results of cell proliferation indicated that the level of RWPE-1 cell proliferation was significantly increased after LPS induction. While miR-181c mimic suppressed the proliferation of RWPE-1 cells. However, the level of cell proliferation was increased when miR-181c and pcDNA-COX-2 were transfected simultaneously in RWPE-1 cells (Figure 5A, B).

Overexpression of miR-181c inhibited inflammatory response in prostate tissue of rats with CNP

Finally, the rats were injected with lentiviruses carrying miR-181c mimic, and the role of miR-181c in CNP rat was then explored. Results showed that the locomotion score and prostate volume of rats were increased after modeling, while the locomotion score and prostate volume of rats injected with miR-181c mimic were decreased (Figure 6A, B). Overexpression of miR-181c reduced the number of inflammatory cells and PCNA-positive cells in prostate tissues (Figure 6C, D). The results of qRT-PCR and western blotting indicated that miR-181c expression was increased and the expression of COX-2 mRNA and protein was decreased after injection of lentivirus carrying miR-181c mimic (Figure 6E). 


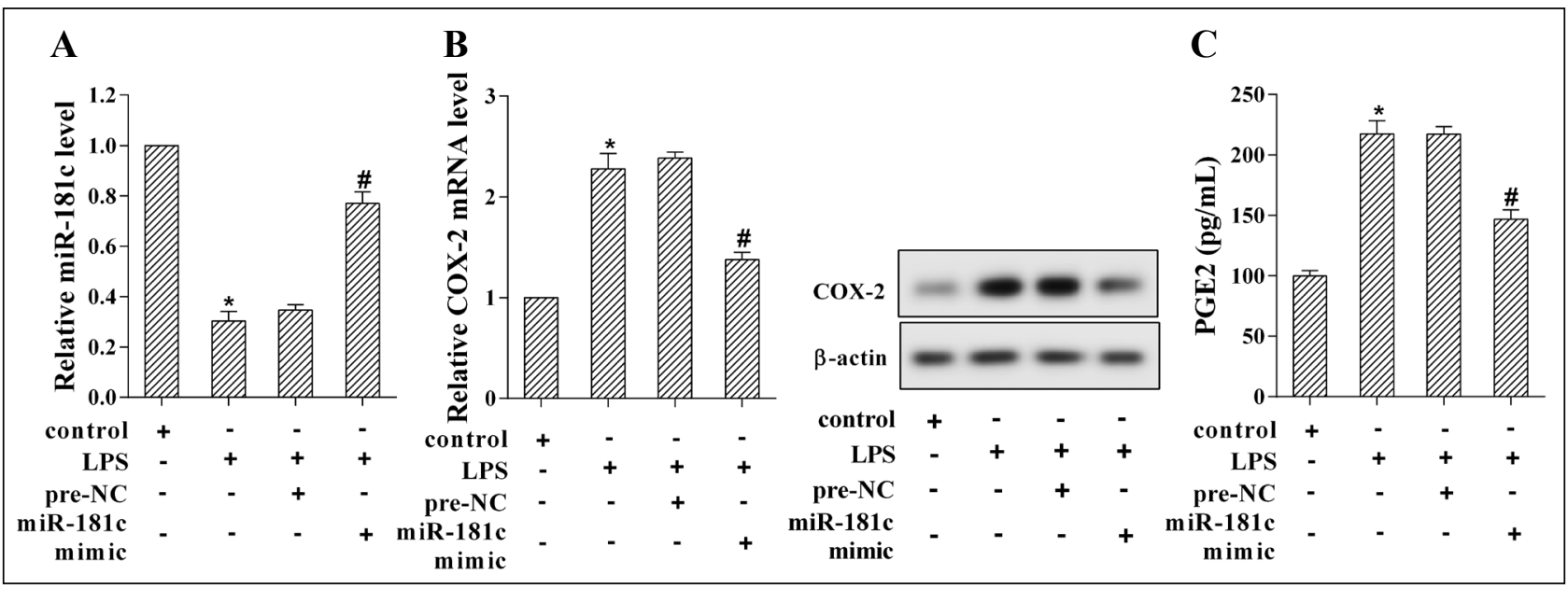

Figure 4. miR-181c mimic or pre-NC were transfected into RWPE-1 cells. Cells were grouped as follows: control, LPS, LPS+pre-NC, and LPS+miR181c mimic group. (A) miR-181c expression was measured with qRT-PCR analysis. (B) The mRNA and protein expression of COX-2 was examined using western blotting. (C) ELISA analysis was applied to detect the concentration of PGE2. ${ }^{*} \mathrm{P}<0.05$ vs. the control group, $\# \mathrm{P}<0.05$ vs. the LPS + preNC group.

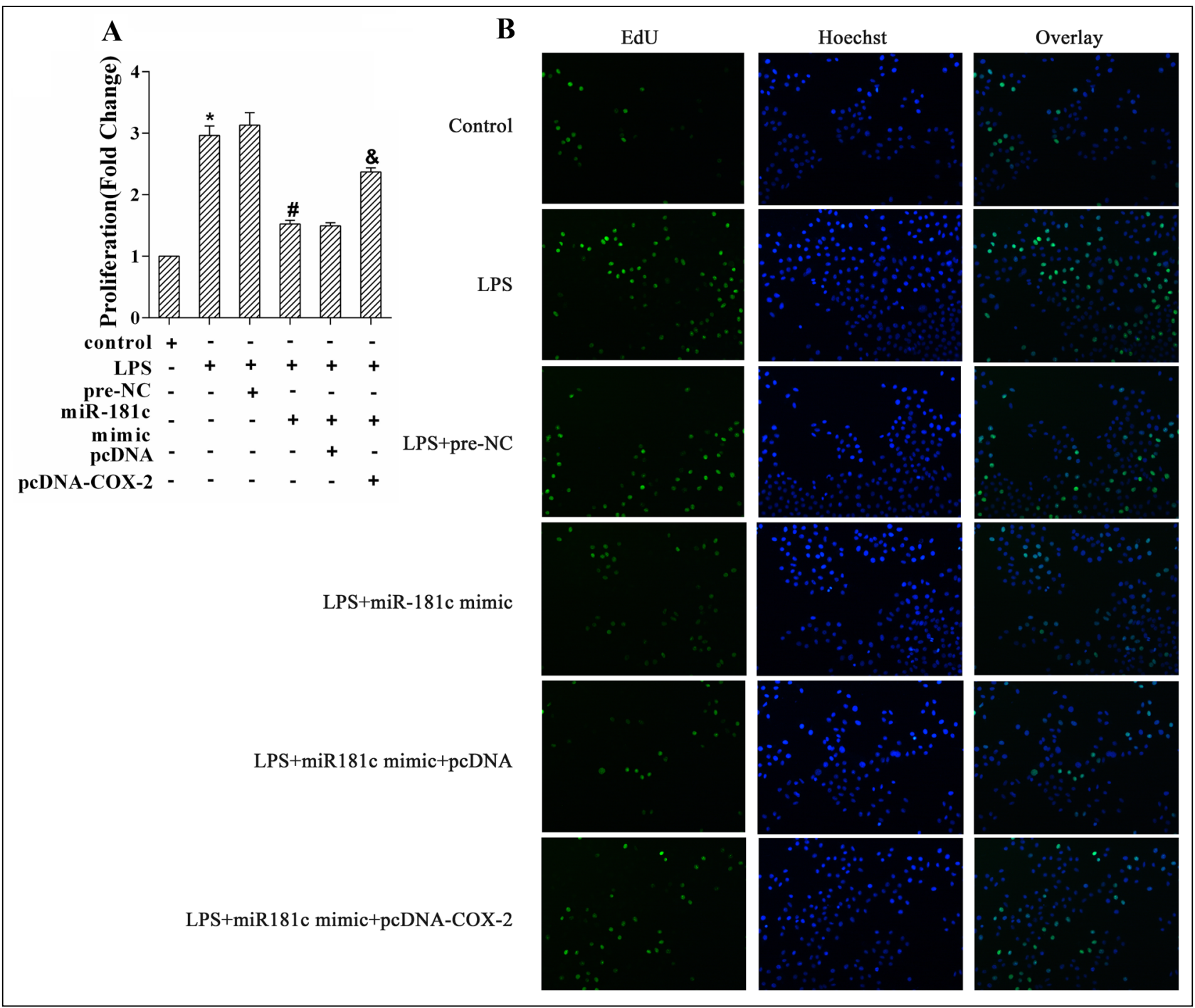

Figure 5. miR-181c mimic, pcDNA-COX-2, and its corresponding negative control (pre-NC and pcDNA) were transfected into RWPE-1 cells. Cells were grouped as follows: control, LPS, LPS + pre-NC, LPS + miR-181c mimic, LPS + miR-181c mimic + pcDNA, LPS + miR-181c mimic + pcDNACOX-2 group. (A, B) MTT assay and EdU assay was employed to measure cell proliferation. ${ }^{*} \mathrm{P}<0.05$ vs. the control group, $\# \mathrm{P}<0.05$ vs. the LPS + pre$\mathrm{NC}$ group, \& $\mathrm{P}<0.05$ vs. the LPS + miR-181c mimic + pcDNA group. 


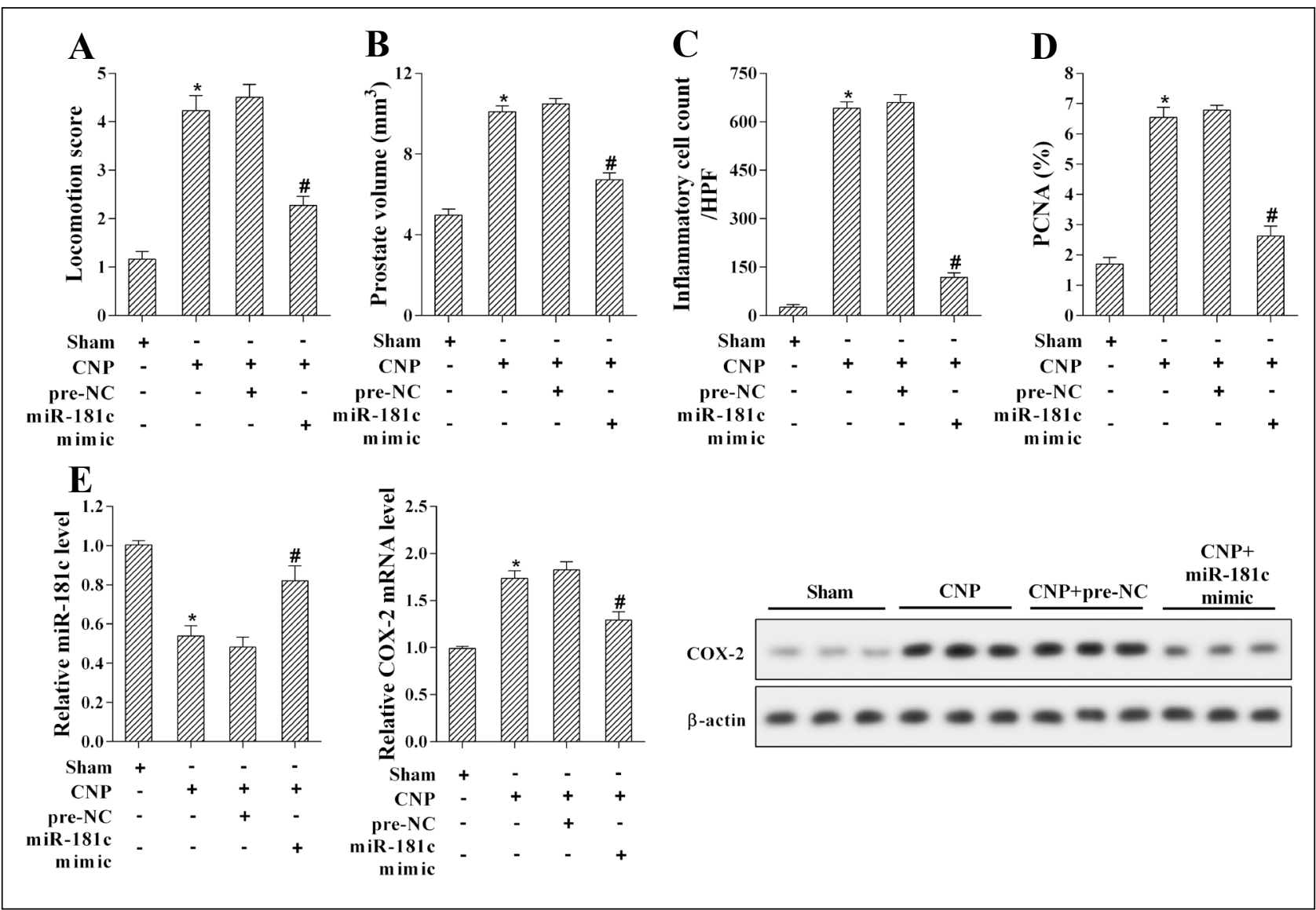

Figure 6. Rats were infected with lentivirus carrying miR-181c mimic or pre-NC. Animals were grouped as follows: Sham, CNP, CNP+preNC, CNP+miR-181c mimic group. (A) Seven days after modeling, the locomotion score was evaluated. (B) Seven days after modeling, rats were sacrificed and prostate tissues were taken. Prostate volume was measured. (C) The average number of inflammatory cells was detected in prostate tissues at high-power fields. (D) Prostatic epithelial cell proliferation was measured in prostate tissues. The relative number of PCNA-positive cells (\%) represented the level of epithelial cell proliferation. (E) qRT-PCR or western blotting was carried out to examine miR-181c and COX-2 expression. $* \mathrm{P}<0.05$ vs. the Sham group, $\# \mathrm{P}<0.05$ vs. the pre-NC group.

\section{Discussion}

$\mathrm{CP} / \mathrm{CPPS}$ is the most widespread type of prostatitis. $\mathrm{CP} /$ CPPS patients will be accompanied by urinary tract symptoms and decreased quality of life. To date, some related mechanisms have been found involved in the occurrence and development of CNP [25-27]. COX-2 is an "early and immediate" gene [28], its main role is to catalyze the production of a group of prostaglandins (PGs) products with different chemical properties, including prostaglandin E2 (PGE2), prostaglandin I2 (PGI2), Prostaglandin F2 $\alpha$ (PGF2 $\alpha$ ), etc. PGE2 is the main catalytic product. The massive synthesis of PGE2 promotes the degranulation of neutrophils to release lysosomes, which intensifies the infiltration of local inflammatory cells and promotes inflammation [29]. In our study, we constructed a CNP rat model induced by carrageenan. This model is similar to some pathological symptoms of clinical CNP patients such as prostate pain, lameness, high increase of prostate volume, increased inflammatory cell and prostate epithelial cell proliferation, indicating that carrageenan-induced CNP rat model can better reflect chronic non-bacterial prostatitis, and providing a reliable theoretical basis for clinical research. In addition, we found that COX-2 was highly expressed in prostate tissues of CP patients. Simultaneously, COX-2 expression was up-regulated in human prostate cells RWPE-1 and CNP rats induced by LPS, which is consistent with previous studies [16,30]. Therefore, elevated COX-2 was a key feature of CNP, which led to an increase in the synthesis of PGE2 and aggravated the inflammatory response in CNP rats.

The prostate is an important accessory gland in men. The prostate includes basal epithelial cells and luminal epithelial cells, as well as rare neuroendocrine cells. The function of prostate epithelium is to secrete prostatic fluid, prostate-specific protein PSA (also known as $\gamma$ semen protein), prostatic acid phosphatase (PAP), etc [31]. Prostatic epithelial cell proliferation plays a vital role in prostate diseases. The level of prostatic epithelial cell proliferation is increased in prostate-related diseases such as CNP [32]. Moreover, inhibition of $\mathrm{COX}-2$ protein can suppress the proliferation of prostate epithelial cells [16]. Our studies have also confirmed this point. Overexpression of COX-2 promoted the proliferation of human prostaglandin epithelial cell RWPE-1. On the contrary, inhibition of COX-2 led to a decrease in the expression of its synthetic product PGE2, simultaneously suppressed prostatic epithelial cell 
proliferation, and reduced carrageenan-induced damage to the prostate tissue of CNP rats. These results show that suppression of prostate epithelial cell proliferation improves chronic non-bacterial prostatitis.

miRNAs can regulate gene expression through a complete or incomplete pairing of their seed sequences with the 3 'untranslated region (3'UTR) of their target genes, which can degrade the mRNA of target genes or suppress the translation of target genes [33, 34]. For example, Ding et al. [35] found that inhibiting miR-146a up-regulated NOS1, which reduced the incidence of erectile dysfunction in patients with CP. In addition, Wang et al. [30] found that up-regulation of miR-141 regulated Keap/ Nrf2 signaling pathway, and reduced the expression of COX-2, the value of locomotion score, eye score, average inflammatory cell count, improving prostatitis. In this study, we found that miR-181c targeted 3'UTR of COX2 to negatively regulate COX-2 mRNA and protein levels. Also, miR-181c overexpression inhibited the proliferation of prostatic epithelial cells, reduced the locomotion score of CNP rats, and inflammatory damage of prostate tissue. These indicate that miR-181c/COX-2 plays a role in CNP. Consequently, in the clinical treatment of chronic abacterial prostatitis in elderly male patients, the expression of miR-181c can be up-regulated to improve prostate pain, dyskinesia, and inflammation.

In conclusion, this study determined that miR-181c expression was decreased in prostate tissues and prostatic epithelial cells, while COX-2 expression was increased. miR-181c inhibits the proliferation of human prostate epithelial cells by negatively regulating COX-2. In vivo experiments, miR-181c overexpression reduced the damage of CNP. This study reveals the mechanism of prostatic epithelial cell proliferation in CNP, which will offer new ideas for the treatment of CNP.

\section{Declarations}

Conflict of interest: The authors declare no conflict of interests.

\section{References}

1. Pontari MA: Chronic prostatitis/chronic pelvic pain syndrome in elderly men: toward better understanding and treatment. Drugs \& aging 2003, 20(15):1111-1125.

2. Krieger J N, Nyberg Jr L, Nickel J C. NIH consensus definition and classification of prostatitis. Jama, 1999, 282(3): 236-237.

3. Fei X, Jin W, Hua S, et al. Prospective study on association of prostatic calcifications with clinical symptoms and results of treatment in men with type III prostatitis. Scientific Reports, 2017, 7(1): 1-6.

4. Nickel J C, Freedland S J, Castro-Santamaria R, et al. Chronic prostate inflammation predicts symptom progression in patients with chronic prostatitis/chronic pel- vic pain. The Journal of urology, 2017, 198(1): 122-128.

5. Magistro G, Wagenlehner F M E, Grabe M, et al. Contemporary management of chronic prostatitis/chronic pelvic pain syndrome. European urology, 2016, 69(2): 286-297.

6. Polackwich A S, Shoskes D A. Chronic prostatitis/chronic pelvic pain syndrome: a review of evaluation and therapy. Prostate Cancer and Prostatic Diseases, 2016, 19(2): 132-138.

7. Pontari M A, Joyce G F, Wise M, et al. Prostatitis. The Journal of urology, 2007, 177(6): 2050-2057.

8. Pontari M A. Chronic prostatitis/chronic pelvic pain syndrome. Urologic Clinics of North America, 2008, 35(1): 81-89.

9. El-Haggar R, Al-Wabli R I. Anti-inflammatory screening and molecular modeling of some novel coumarin derivatives. Molecules, 2015, 20(4): 5374-5391.

10. Stables M J, Newson J, Ayoub S S, et al. Priming innate immune responses to infection by cyclooxygenase inhibition kills antibiotic-susceptible and-resistant bacteria. Blood, The Journal of the American Society of Hematology, 2010, 116(16): 2950-2959.

11. Bhardwaj A, Kaur J, Wuest M, et al. In situ click chemistry generation of cyclooxygenase- 2 inhibitors. Nature Communications, 2017, 8(1): 1-14.

12. Khanapure S P, Garvey D S, Janero D R, et al. Eicosanoids in inflammation: biosynthesis, pharmacology, and therapeutic frontiers. Current topics in medicinal chemistry, 2007, 7(3): 311-340.

13. Zheng Y, Comaills V, Burr R, et al. COX-2 mediates tumorstromal prolactin signaling to initiate tumorigenesis. Proceedings of the National Academy of Sciences, 2019, 116(12): 5223-5232.

14. Yuan Z, Liu X, Deng W, et al. Correlation study of chronic nonbacterial prostatitis with the levels of COX-2 and PGE2 in prostatic secretion. International urology and nephrology, 2014, 46(10): 1871-1875.

15. Jeon S H, Zhu G Q, Kwon E B, et al. Extracorporeal shock wave therapy decreases COX-2 by inhibiting TLR4-NFKB pathway in a prostatitis rat model. The Prostate, 2019, 79(13): 1498-1504.

16. Xu X, Hou J, Lv J, et al. Overexpression of IncRNA GAS5 suppresses prostatic epithelial cell proliferation by regulating COX-2 in chronic non-bacterial prostatitis. Cell Cycle, 2019, 18(9): 923-931.

17. Lee R C, Feinbaum R L, Ambros V. The C. elegans heterochronic gene lin-4 encodes small RNAs with antisense complementarity to lin-14. cell, 1993, 75(5): 843-854.

18. Kotaja N. MicroRNAs and spermatogenesis. Fertility and sterility, 2014, 101(6): 1552-1562.

19. Sur S, Steele R, Shi X, et al. miRNA-29b inhibits prostate tumor growth and induces apoptosis by increasing bim expression. Cells, 2019, 8(11): 1455.

20. Wang X, Gao Y, Tian N, et al. Astragaloside IV improves renal function and fibrosis via inhibition of miR-21-induced podocyte dedifferentiation and mesangial cell activation in diabetic mice. Drug design, development and therapy, 2018, 12: 2431.

21. Ayala-Ortega E, Arzate-Mejía R, Pérez-Molina R, et al. 
Epigenetic silencing of miR-181c by DNA methylation in glioblastoma cell lines. BMC cancer, 2016, 16(1): 226.

22. Ngalame N N O, Tokar E J, Person R J, et al. Aberrant microRNA expression likely controls RAS oncogene activation during malignant transformation of human prostate epithelial and stem cells by arsenic. Toxicological sciences, 2014, 138(2): 268-277.

23. Fryczkowski M, Bułdak R J, Hejmo T, et al. Circulating levels of omentin, leptin, VEGF, and HGF and their clinical relevance with PSA marker in prostate cancer. Disease markers, 2018, 2018.

24. Ribeiro D L, Pinto M E, Rafacho A, et al. High-fat diet obesity associated with insulin resistance increases cell proliferation, estrogen receptor, and PI3K proteins in rat ventral prostate. Journal of andrology, 2012, 33(5): 854865.

25. Meng L Q, Yang F Y, Wang M S, et al. Quercetin protects against chronic prostatitis in rat model through NF$\kappa \mathrm{B}$ and MAPK signaling pathways. The Prostate, 2018, 78(11): 790-800.

26. Pontari M A, Ruggieri M R. Mechanisms in prostatitis/ chronic pelvic pain syndrome. The Journal of urology, 2008, 179(5S): S61-S67.

27. Liu X, Ran X, Riaz M, et al. Mechanism Investigation of Tagetes patula L. against Chronic Nonbacterial Prostatitis by Metabolomics and Network Pharmacology. Molecules, 2019, 24(12): 2266.

28. Chandrasekaran B, Pal D, Kolluru V, et al. The chemopreventive effect of withaferin $A$ on spontaneous and inflammation-associated colon carcinogenesis models.
Carcinogenesis, 2018, 39(12): 1537-1547.

29. Zhou S, Zhou Z, Ding K, et al. DREAM-in-CDM approach and identification of a new generation of anti-inflammatory drugs targeting mPGES-1. Scientific reports, 2020, 10(1): 1-9.

30. Wang L L, Huang Y H, Yan C Y, et al. N-acetylcysteine ameliorates prostatitis via miR-141 regulating Keap1/ Nrf2 signaling[J]. Inflammation, 2016, 39(2): 938-947.

31. Zhang D, Jeter C, Gong S, et al. Histone 2B-GFP labelretaining prostate luminal cells possess progenitor cell properties and are intrinsically resistant to castration. Stem cell reports, 2018, 10(1): 228-242.

32. Dang T, Liou G Y. Macrophage cytokines enhance cell proliferation of normal prostate epithelial cells through activation of ERK and Akt. Scientific reports, 2018, 8(1): 1-9.

33. Tran A T, Chapman E M, Flamand M N, et al. MiR-35 buffers apoptosis thresholds in the C. elegans germline by antagonizing both MAPK and core apoptosis pathways. Cell Death \& Differentiation, 2019, 26(12): 2637-2651.

34. Yu B, Lv X, Su L, et al. MiR-148a functions as a tumor suppressor by targeting CCK-BR via inactivating STAT3 and Akt in human gastric cancer. PLoS One, 2016, 11(8): e0158961.

35. Ding J, Tang Y, Tang Z, et al. A Variant in the precursor of microRNA-146a is responsible for development of erectile dysfunction in patients with chronic prostatitis via targeting NOS1. Medical Science Monitor: International Medical Journal of Experimental and Clinical Research, 2017, 23: 929.

Cite this article as: Xu X, Huang Y, Hou J, et al. miR-181c inhibits prostatic epithelial cell proliferation caused by chronic non-bacterial prostatitis through down-regulating COX-2[J]. Aging Pathobiology and Therapeutics, 2020, 2(4): 210-218. 